\title{
DOUBLE-LINED SPECTROSCOPIC BINARY STARS IN THE RAVE SURVEY
}

\author{
G. Matijevič ${ }^{1}$, T. Zwitter ${ }^{1,2}$, U. Munari ${ }^{3}$, O. Bienaymé ${ }^{4}$, J. Binney ${ }^{5}$, J. Bland-Hawthorn ${ }^{6}$, C. Boeche ${ }^{7}$, R. Campbell ${ }^{8}$, \\ K. C. Freeman ${ }^{9}$, B. Gibson ${ }^{10}$, G. Gilmore ${ }^{11}$, E. K. Grebel ${ }^{12}$, A. Helmi ${ }^{13}$, J. F. Navarro ${ }^{14}$, Q. A. Parker ${ }^{15}$, G. M. Seabroke ${ }^{16}$, \\ A. Siebert ${ }^{4}$, A. Siviero ${ }^{3,7}$, M. Steinmetz ${ }^{7}$, F. G. Watson ${ }^{17}$, M. Williams ${ }^{7}$, And R. F. G. Wyse ${ }^{18}$ \\ ${ }^{1}$ Faculty of Mathematics and Physics, University of Ljubljana, Ljubljana, Slovenia; gal.matijevic@ @mf.uni-lj.si \\ ${ }^{2}$ Center of Excellence SPACE-SI, Ljubljana, Slovenia \\ ${ }^{3}$ INAF Osservatorio Astronomico di Padova, Asiago, Italy \\ ${ }^{4}$ Observatorie de Strasbourg, Strasbourg, France \\ ${ }^{5}$ Rudolf Pierls Center for Theoretical Physics, University of Oxford, Oxford, UK \\ ${ }^{6}$ Sydney Institute for Astronomy, School of Physics, University of Sydney, Sydney, Australia \\ ${ }^{7}$ Astrophysikalisches Institut Potsdam, Potsdam, Germany \\ ${ }^{8}$ Western Kentucky University, Bowling Green, KY, USA \\ ${ }^{9}$ RSAA, Australian National University, Camberra, Australia \\ ${ }^{10}$ University of Central Lancashire, Preston, UK \\ ${ }^{11}$ Institute of Astronomy, Cambridge, UK \\ 12 Astronomisches Rechen-Institut, Zentrum für Astronomie der Universität Heidelberg, Heidelberg, Germany \\ ${ }^{13}$ Kapteyn Astronomical Institute, University of Groningen, Groningen, The Netherlands \\ ${ }^{14}$ University of Victoria, Victoria, Canada \\ ${ }^{15}$ Macquarie University, Sydney, Australia \\ ${ }^{16}$ e2v Centre for Electronic Imaging, Planetary and Space Sciences Research Institute, The Open University, Walton Hall, Milton Keynes, UK \\ ${ }_{17}$ Anglo-Australian Observatory, Sydney, Australia \\ ${ }^{18}$ John Hopkins University, Baltimore, MD, USA \\ Received 2010 March 3; accepted 2010 May 17; published 2010 June 8
}

\begin{abstract}
We devise a new method for the detection of double-lined binary stars in a sample of the Radial Velocity Experiment (RAVE) survey spectra. The method is both tested against extensive simulations based on synthetic spectra and compared to direct visual inspection of all RAVE spectra. It is based on the properties and shape of the crosscorrelation function, and is able to recover $\sim 80 \%$ of all binaries with an orbital period of order 1 day. Systems with periods up to $1 \mathrm{yr}$ are still within the detection reach. We have applied the method to 25,850 spectra of the RAVE second data release and found 123 double-lined binary candidates, only eight of which are already marked as binaries in the SIMBAD database. Among the candidates, there are seven that show spectral features consistent with the RS CVn type (solar type with active chromosphere) and seven that might be of W UMa type (over-contact binaries). One star, HD 101167, seems to be a triple system composed of three nearly identical G-type dwarfs. The tested classification method could also be applicable to the data of the upcoming Gaia mission.
\end{abstract}

Key words: binaries: spectroscopic - methods: data analysis - surveys

\section{INTRODUCTION}

Double-lined spectroscopic binaries, and in particular their eclipsing variants (EB-SB2s), are important astrophysical testbeds that provide a wealth of constraints on stellar models. EB-SB2s are the primary provider of accurate stellar masses and radii. When coupled with accurate atmospheric temperatures, the orbital solution of EB-SB2s geometrically fixes distance with great accuracy, becoming a critical testbed even for Hipparcos astrometric parallaxes (e.g., the case of the Pleiades cluster; Munari et al. 2004; van Leeuwen 2007, and references therein). If the components of a binary were born together, then they lie on the same isochrone. This constrains their metallicity and stellar models that reproduce them, including recent refinements such as the efficiency of overshooting and the role of atmospheric sedimentation of heavy elements (e.g., Tomasella et al. 2008). Torres et al. (2009) provide a recent and updated review of astrophysical results based on the study of binaries.

The Radial Velocity Experiment (RAVE) is an ongoing multifiber spectroscopic survey based on the UK Schmidt Telescope at the Anglo Australian Observatory. With a goal of observing 1 million stars, the survey has so far gathered more than 400,000 spectra in the magnitude range between $9<I_{\mathrm{C}}<$ 13. The wavelength range of spectra covers the near-infrared (near-IR) interval from $8400 \AA$ to $8800 \AA$ with a resolving power of $\sim 7500$, typically with a high signal-to-noise ratio $(\mathrm{S} / \mathrm{N}$; mean value of 45). This range is virtually free from any telluric lines. The Doppler shift of the lines permits us to measure the radial velocity to a precision of $1.3 \mathrm{~km} \mathrm{~s}^{-1}$, and several prominent metallic and hydrogen spectral lines make it possible to derive accurate stellar atmospheric parameters and chemical composition (see Zwitter et al. 2004; Boeche et al. 2010 for more details). So far two data releases have been published (Steinmetz et al. 2006; Zwitter et al. 2008, hereafter Z08), and a third one will soon be released (A. Siebert et al. 2010, in preparation).

The primary goal of the RAVE survey is to measure precise radial velocities and atmospheric parameters of up to a million normal single stars with known proper motions and photometric data. The atmospheric parameters are used to infer the absolute magnitude and to estimate the distance to the targets (Breddels et al. 2010; T. Zwitter et al. 2010, in preparation). This in turn fixes all six phase-space coordinates and Galactic orbits. When coupled with information on metallicity and chemical abundances (which RAVE also provides), the RAVE survey is well suited to investigate Galactic structure and dynamics (Smith et al. 2007; Seabroke et al. 2008; Siebert et al. 2008; Veltz et al. 2008).

The unbiased sample of input stars selected for RAVE also includes, of course, a minority of peculiar and spectroscopic binary stars. The ability of RAVE spectra to identify and 
properly characterize spectra with special features has already been demonstrated by the study of luminous blue variable supergiants in the Large Magellanic Cloud (Munari et al. 2009) and diffuse interstellar bands over the RAVE wavelength range (Munari et al. 2008). Double-lined binary stars are not specifically tackled by the RAVE main analysis pipeline. The aim of this paper is to discuss a tool parallel to the main pipeline to identify double-lined binary star candidates (and to distinguish them from other types of peculiar stars, in particular those showing emission-line cores), and to derive the radial velocity and atmospheric parameters of both components. The analysis is carried out on the RAVE second data release. Singlelined binaries collected from repeated observations will be treated in a separate paper. Section 2 discusses the SB2 detection method, based on the shape of the cross-correlation function (CCF). In Section 3, we evaluate the performance of our method on a sample of synthetic binary spectra. Two tables in Section 4 list all the binary candidates we have discovered among RAVE stars from the second data release, along with radial velocities and effective temperatures of both components.

\section{CLASSIFICATION OF PECULIAR SPECTRA}

The identification of all peculiar and SB2 spectra in the RAVE survey is relevant because (1) it cleans the survey products from potentially faulty results, (2) it offers a list of objects that are interesting per se and worthy of further as well as individual consideration, and (3) it will eventually allow for a population study of those types of objects. The large (and continuously growing) number of spectra recorded by the RAVE survey makes it impossible to evaluate all of them by hand. The identification of peculiar and SB2 spectra has to be carried out by automated procedures. To gain specific experience and to provide a comparison ground for the results of the automated procedure, we have nevertheless performed an eye inspection on each of the $\sim 25,000$ spectra included in the second RAVE data release.

For the purpose of identification of peculiar and SB2 spectra, we have adopted a method based on the properties of the $\mathrm{CCF}$ between the observed spectrum $o(\lambda)$ and a synthetic template spectrum $t(\lambda)$,

$$
\int_{R} o(\lambda) t(\bar{\lambda}-\lambda) d \lambda
$$

with integration covering the whole spectral range $R$ and the synthetic spectrum taken from the library of Munari et al. (2005), the same as adopted for the main RAVE $\chi^{2}$ analysis pipeline. The procedure works by examining several properties of the observed spectra and the CCF in a few steps. As an input it requires a flux-normalized observed spectrum, its $\mathrm{S} / \mathrm{N}$ value, and a rough estimate of the effective temperature. The latter two values are already provided by the main RAVE analysis pipeline.

We have also tested other numerical methods that are commonly used for the purpose of classification-artificial neural networks, support vector machines, and principal component analysis. None of those methods worked well for our purposes, most likely due to the overwhelming number of different morphological features that are present in the observed spectra, which are extremely hard to efficiently represent in a training sample. For this reason we focused on the cross-correlation method, which is detailed in the following section.

\subsection{Classification Procedure}

The goal of the classification pipeline is to separate different types of spectra and to discover spectra with systematic or other observational errors. It is required that the value of the $\mathrm{S} / \mathrm{N}$ is greater than 13. That limit was set in Z08 where it was shown that the estimation of atmospheric parameters for spectra at lower $\mathrm{S} / \mathrm{N}$ becomes unreliable and only the major spectral lines are still distinguishable. It seems reasonable to assume that classification would also become unreliable below that limit.

Next, the minimal and maximal flux values are verified. If the minimal value (of any single pixel) is negative, the spectrum is rejected since it has clearly undergone some problems in background subtraction. On the other hand, the limits on maximal values are more problematic because rejecting spectra with emission lines is not our goal. Taking that into account, we set the upper limit to 1.5 by inspecting the normalized flux distribution of a large number of spectra. It showed that less than $1 \%$ of the spectra have their maximum flux above that value, mostly as a result of an artifact. Such spectra are not immediately excluded but are flagged as potential cosmic-ray hit candidates. Emission-line objects (see Figure 1(h)) are identified later.

In the next step, several properties of the CCF between the observed spectrum and the synthetic template are calculated and evaluated. The template spectrum is interpolated based on the library of synthetic spectra by Munari et al. (2005). We always construct the CCF using the same template. In our experience, this allows for an easier classification of unusual spectra (e.g., coronal emission, binaries, etc.) because in those cases the resulting CCF is not influenced by the erroneous formally best-matching normal star template, calculated by the parameter estimation pipeline. For the template, we use the average case of a dwarf with $T_{\text {eff }}=5800 \mathrm{~K}, \log (g)=4.4$, and $\mathrm{M} / \mathrm{H}=0.0$ (i.e., close to solar values). The calculation of the $\mathrm{CCF}$ is done with the same template for all observed spectra. This means that the peaks of CCFs of hotter stars, for example, will be weaker but it is of no concern since we are only interested in the particular shape of the CCF and not in its strength. For the same reason, the exact values of the selected atmospheric parameters of the template spectrum are not important and the properties of the CCF do not depend strongly on the values of those parameters. The observed spectrum is then re-binned to the template's wavelength range for an easier calculation. Z08 also showed that the projected rotational velocity $(v \sin i)$ is not recoverable for a slowly rotating star, while the amount of $\alpha$-enhancement $([\alpha / \mathrm{Fe}])$ is unreliable and cannot be trusted for individual objects. Therefore, both parameters are set to zero. Another reason why a more careful selection of rotational velocity is not important is the fact that the width of the core of the $\mathrm{CCF}$ is much wider than the error of the typical rotational velocity estimate.

The wings of the CCF contain information about the oscillations of the spectral continuum. By comparing the asymptotic values of the wings, it is possible to eliminate all spectra that have uneven continuum and are unsuitable for modeling. Figure 1(b) shows a relatively high $\mathrm{S} / \mathrm{N}$ spectrum with a strongly oscillating continuum. While the left wing converges to zero as it does in the case of a well-behaved single star spectrum, the right one does not. The difference of the two levels is a useful method for the detection of badly normalized spectra.

Distinguishing between different types of stellar objects (e.g., binary stars, emission stars, fast rotators) is harder than detecting spectra plagued by observational and systematic errors. Spectral features in the former might look similar in some cases (split 


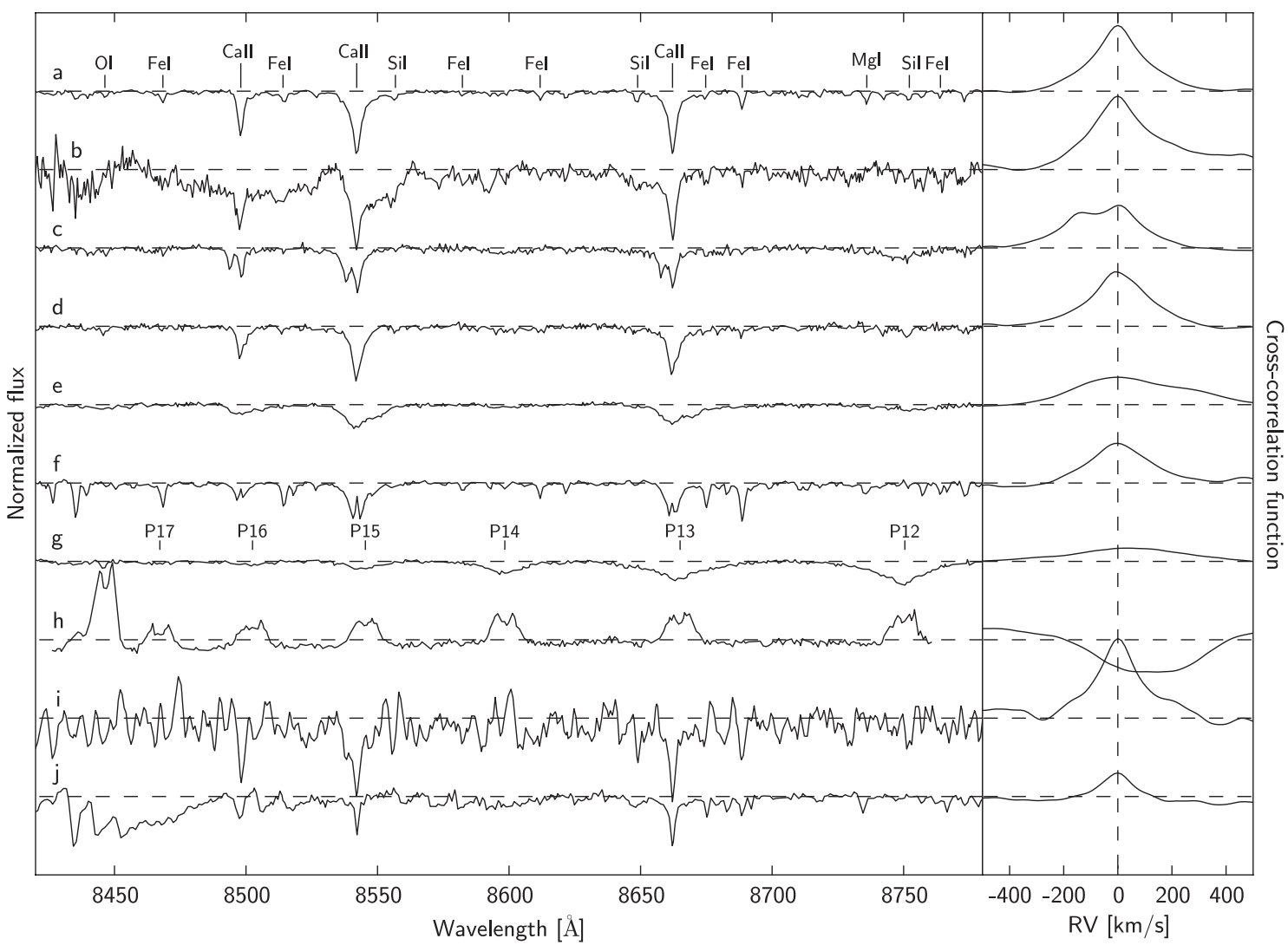

Figure 1. Spectra and CCFs of various stellar objects: (a) well-behaved spectrum of a G2V star, (b) spectrum with bad continuum fit, (c) wide SB2 binary candidate, (d) blended SB2 binary candidate, (e) W UMa type contact binary candidate, (f) star with chromospheric emission, (g) B5V star, (h) Be star, (i) carbon star, and (j) cold star with molecular bands $(<3500 \mathrm{~K})$. The dashed threshold lines are positioned at value 1 for spectra plots and at value 0 for CCF plots. All vertical scales are equal.

major lines in spectra of stars with chromospheric emission and double-lined binary spectra, for example). Counting the number of peaks of the CCFs is extremely efficient in the detection of potential SB2 binaries (Figure 1(c)). If the CCF has more than one central peak, the spectrum is clearly doublelined and probably comes from an SB2. In the case of blended SB2-like spectra, the CCF does not show two distinct peaks but is still asymmetric (Figure 1(d)). Unfortunately, CCFs of spectra of stars with chromospheric emission (Figure 1(f)) look similar to the previous case. Discriminating between those two classes is possible by measuring the width of the CCF and the level of asymmetry. The width of the CCF for a spectrum showing chromospheric emission cores is equal to that of the underlying star without the emission-line cores. The level of asymmetry is calculated by comparing the area under the left and right half of the CCF. It turns out that CCFs of blended binary candidates are wider and more asymmetric than those of stars with emission-line cores. The border values were set by inspecting a visually classified sample of both types. Stars with emission-line cores usually have stronger narrow metallic lines that help to distinguish between both types. In cases where spectral lines of an SB2 candidate are too blended, the detection of binarity is impossible. Further details on this topic are described in the following section.

Wide and shallow spectral lines are a signature of hot stars, where the otherwise dominant $\mathrm{Ca}$ II triplet is weak or absent and lines from the hydrogen Paschen series dominate. The CCF between such a spectrum and a template spectrum of a colder dwarf is wide and with low amplitude (Figure $1(\mathrm{~g})$ ). Since lines get wider and shallower with increasing temperature, it becomes increasingly difficult to recognize SB2 binary candidates among early A-type and hotter stars, even if they are observed at quadratures and close to edge-on inclinations. Fortunately, the fraction of hot stars among the high galactic latitude field RAVE stars is almost negligible. Relatively low amplitude and very wide CCFs are also observed in spectra of contact binaries. Most of the lines in such spectra display very little contrast with the underlying continuum: in addition to their intrinsic shallowness they are further widened by rapid rotation (Figure 1(e)). They too can be recognized by inspecting the asymmetry of the CCF. Fast rotating stars are another type of object that shows similar CCFs. According to Glebocki \& Stawikowski (2000), the average projected rotational velocity becomes significant (greater than $30 \mathrm{~km} \mathrm{~s}^{-1}$ on average) for stars earlier than type $\mathrm{F} 5$, setting a limit at $T_{\text {eff }}=6600 \mathrm{~K}$. If some wide-lined spectrum that does not have a clear two-peaked CCF has a temperature above this limit, it is considered to be a fast rotator rather than a blended binary.

Peculiar types of stars are harder to classify since spectral features of such objects span a wide range of appearances. CCFs of special cases (Figures 1(h)-(j)) all look different from normal single star spectra. All such peculiar objects were flagged and later eye-checked to avoid any misclassification. A detailed description of the classification procedure along with the computer code is available upon request from the authors.

\section{SB2 DETECTION SIMULATION}

In this section, we explore which parameters have the largest impact on the probability of detection of an SB2 or a peculiar 
star spectrum in the RAVE spectra sample. We also determine the limits of parameter space beyond which their detection is no longer possible.

\subsection{Synthetic Sample}

It is desired that the synthetic sample mimics the observed one as closely as possible. We expect that metallicity $([\mathrm{M} / \mathrm{H}])$ and barycentric radial velocity are both distributed the same way for binary stars as they are for single stars, with the latter values measured by $\mathrm{Z} 08$. The similarity between the metallicity distribution among single and binary stars is discussed by Latham (2003). Additionally, we expect that both components should have roughly the same chemical composition and are both of the same age.

Although in the RAVE survey the number of dwarfs and giants is roughly similar, we assumed that only main-sequence binary stars are likely to be double-lined. For binaries of intermediate masses, it is highly unlikely to find one consisting of two giant components. There are two reasons for this: (1) to reach the giant stage at the same time, two stars must be almost equally massive (to within 1\%) and (2) the lifespan of a giant is much shorter than the lifespan of a dwarf. Combining both criteria makes the probability of finding a giant-giant SB2 very small. Additionally, the morphology of a spectrum of a giant star is similar to a spectrum of a dwarf star with similar effective temperature. So even if we would be dealing with an SB2 spectrum of two giants, the classification method should not have any trouble recognizing that.

As a starting point for constructing a synthetic binary spectrum we took a distribution of temperature, metallicity, and radial velocity for single star dwarfs where we only included spectra with $\mathrm{S} / \mathrm{N}>20$ and $\log (g)>3.5$ from the observed sample of 222,563 stars (from the internal release not yet publicly available) that had been previously confirmed as normal single stars by the classification method. After drawing random picks for the first star's temperature $T_{\text {eff, } 1}$ and $[\mathrm{M} / \mathrm{H}]$ from those distributions both values were randomly varied for typical errors of both measurements, $\sigma_{T}=300 \mathrm{~K}$ and $\sigma_{[\mathrm{M} / \mathrm{H}]}=0.2$. This was done in order to make both distributions smoother. Using the two values, we found a complete set of parameters (mass, radius, and $I$ magnitude) for the first star from a Yonsai-Yale isochrone (Yi et al. 2001) of appropriate iron abundance and an age of 200 Myr. This particular age was chosen so that all stars are already settled on the main sequence and the hotter ones still did not have time to turn to giants, setting the corresponding upper mass cutoff at approximately 3 solar masses. The age dependence of the stellar parameters for the stars on the main sequence can be neglected and the selection of the age of the isochrone is not very important. Similarly, we also neglected the minor effect of $\alpha$-enhancement and its value was kept fixed at zero during the calculation of all spectra. This provides us with all the necessary parameters for the construction of the synthetic spectrum of the first component. Now we randomly select a luminosity ratio $\eta$, which is defined as

$$
\eta=10^{\frac{2}{5}\left(I_{1}-I_{2}\right)}
$$

where $I_{1}$ and $I_{2}$ are the brighter star's and the fainter star's $I$ magnitudes, respectively. This ratio is selected from an interval of $[0,1]$. From there on it is straightforward to find the position on the same isochrone with a matching $I$ magnitude of the second component. Distributions of stellar masses, radii, mass ratios, and luminosity ratios in the final sample are shown in Figure 2.
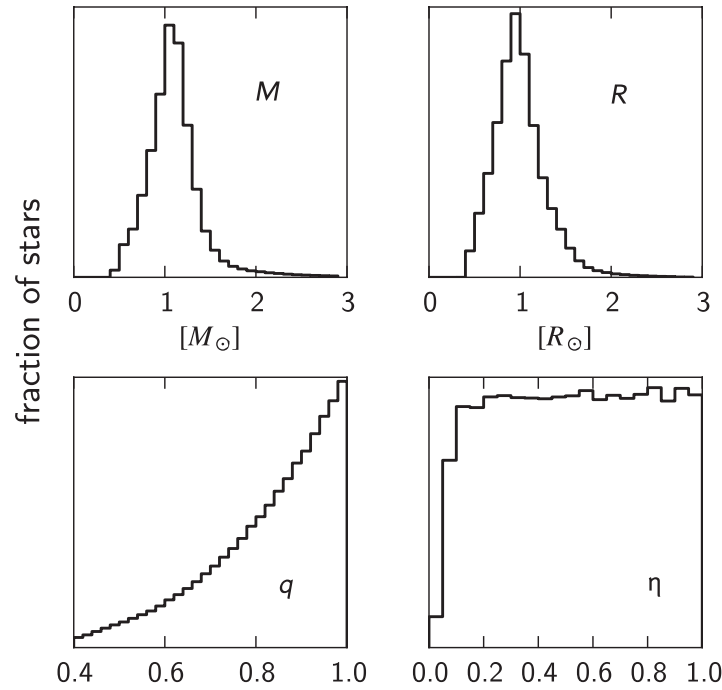

Figure 2. Distributions of stellar masses $(M)$, radii $(R)$, mass ratios $(q)$, and luminosity ratios $(\eta)$ of synthetic binary stars. The distribution of luminosity ratios is flat except for very small values where the systems could not be modeled because of the consequently large temperature difference.

The orbital period was chosen randomly from a distribution fitted to observations in Duquennoy \& Mayor (1991),

$$
f(\log P)=\frac{d N}{d \log P}=C \exp \left\{\frac{-(\log P-\overline{\log P})^{2}}{2 \sigma_{\log P}^{2}}\right\},
$$

where $\overline{\log P}=4.8$ and $\sigma_{\log P}=2.3$ and $P$ is in days. A similar distribution was also found in numerical simulations of star cluster evolution by Bate (2009). A limit on the short period was selected at 0.2 day, because the period distribution for eclipsing binary stars in Malkov et al. (2006) shows a strong cutoff there. On the longer end, we took a generous limit of two years. At such long orbital periods, the orbital velocities become so small that the fraction of detectable binaries becomes negligible. For a binary system of two solar twins on a circular orbit, the maximal velocity amplitude is equal to $30 \mathrm{~km} \mathrm{~s}^{-1}$ for a period of two years. Knowing the orbital period, the semimajor axis and orbital velocities were calculated using Kepler's third law. We assumed that all systems have circular orbits. This only holds true for very short period systems $(P<10$ days). The eccentricity of systems with $P<500$ days can be significant with a mean of about 0.3 , according to Duquennoy \& Mayor (1991). Nevertheless, the differences of orbital velocities of such systems compared to the velocities of similar systems with circular orbits are small in most cases, justifying the upper assumption. The orbital phase and orbital inclination to the line of sight were selected randomly.

The rotational velocities of both stars were calculated by assuming that the stars co-rotate with the system's orbital rotation. The minimal rotational velocity was set at $20 \mathrm{~km} \mathrm{~s}^{-1}$, similar to the value typically obtained by RAVE's parameter estimation pipeline for slowly rotating single stars. While this value is unrealistically high, it simulates the marginally lower resolving power of the observed spectra compared to the synthetic ones, where the difference is easily compensated for by slightly wider lines of faster rotators. Some of the close binary systems were removed from the simulation since their rotational velocity exceeded the highest velocity supported by the spectra library from Munari et al. (2005). The overall number of such 


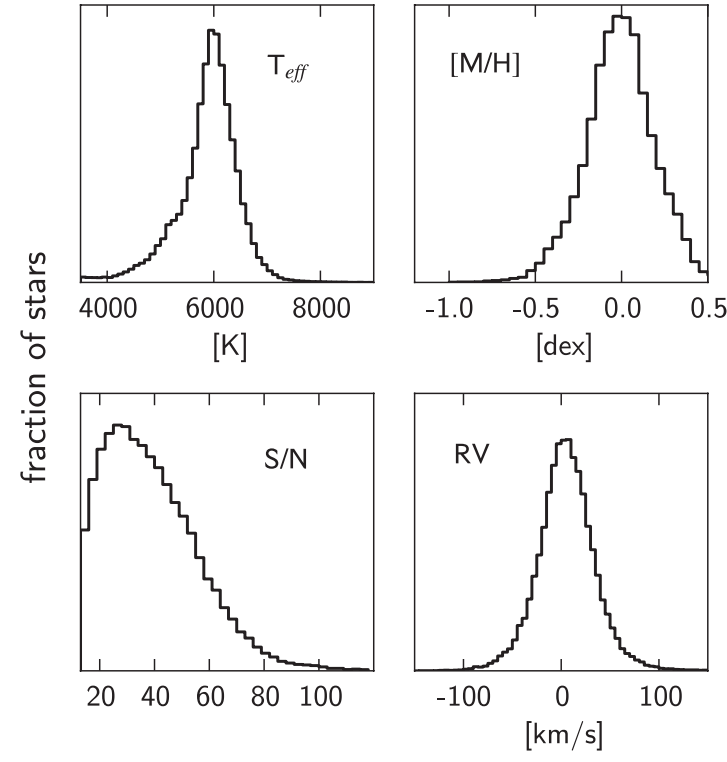

Figure 3. Observed distributions for temperature, metallicity, S/N, and barycentric radial velocity for single stars from which the simulated parameters for synthetic binaries are drawn.

systems is very small and their omission should not affect the end results of the simulation.

Synthetic spectra were then scaled according to the luminosity ratio $\eta$, Doppler-shifted to their projected orbital velocities, summed and normalized, and finally, the barycentric radial velocity drawn from the distribution shown in Figure 3 was applied. To simulate the observational errors, Gaussian noise was added to the binary spectrum according to the randomly selected value of $\mathrm{S} / \mathrm{N}$ from the distribution also shown in Figure 3. Errors in the normalization of the continuum were simulated by introducing five cosine functions with random phases, frequencies, and amplitudes matching typical variations in observed spectra. We generated $10^{6}$ such binary spectra that were then classified with the method described in the previous section.

\subsection{Simulation Results}

One of the results yielded by the simulations is the distribution of system periods at which the binarity is detectable. The limitations on the short- and long-period ends come from two different factors. The spectral lines of short-period binaries are widened because of the co-rotation of both components. Another reason for the widening is velocity smearing during the exposure which becomes significant only for the systems with the shortest periods ( $P<1$ day). Combining both effects can blend the lines of even a relatively well-separated system, making detection more difficult. On the long-period end, the detection probability is affected by the limited resolving power of the spectrograph and the fact that binarity detection strongly depends on the noticeable double peaks of the relatively wide $\mathrm{Ca}$ II triplet lines. For example, a binary system of solar twins orbiting around each other on a circular orbit with a period of one year and seen along a line of sight close to orbital plane is just on the threshold of detection when observed at a quarter phase.

Figure 4 compares the distribution of the input binary systems and those recovered by our analysis. The dashed lines show the distribution of periods given in Equation (3), while the shaded gray histogram shows the input period distribution. The systems with the shortest periods are missing partly because it was not

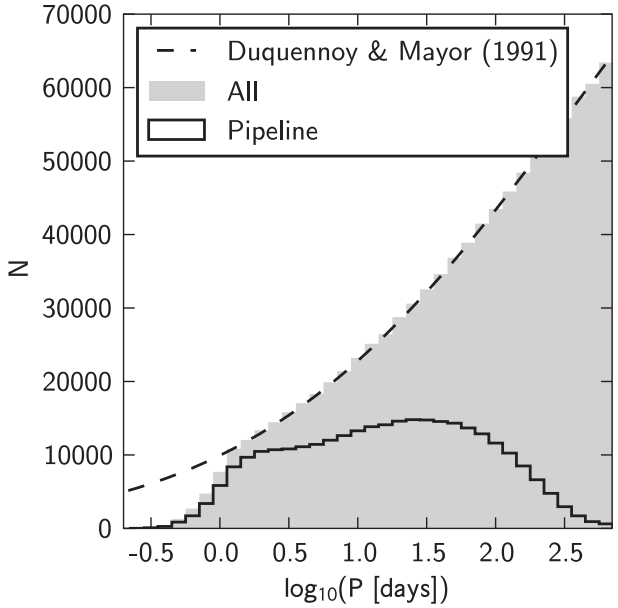

Figure 4. Initial distribution of orbital periods and the distribution of periods of detected systems. The dashed line shows the predicted distribution from the literature given in Equation (3). The shaded gray histogram shows the actual initial distribution of the simulation without the systems with very short periods. The black solid histogram shows the number of detected systems in the simulation.

possible to model spectra of fast rotating cool stars and partly because we removed all systems that exhibited Roche overflow, i.e., in which the stellar radii became greater than the radius of the Roche lobe $R_{L}$ given by Eggleton (1983),

$$
\frac{R_{L}}{a} \approx \frac{0.49 q^{2 / 3}}{0.6 q^{2 / 3}+\ln \left(1+q^{1 / 3}\right)},
$$

where $a$ denotes the semimajor axis and $q$ denotes the mass ratio. The solid line in Figure 4 shows the number of systems that were confirmed as binaries by our classification method. The number of modeled systems is small below $P \leqslant 1$ day and predictions there should not be trusted. The detection ratio becomes very high for systems with periods between 1 and 10 days. For systems with periods between 10 and 100 days, the detection rate is still significant. For $P \geqslant 100$ days the number of recovered binaries rapidly drops, becoming negligible at $1 \mathrm{yr}$.

The dependence of the ratio between the number of properly classified and all binary systems (detection ratio) on various parameters is shown in Figure 5. For all synthetic spectra the classification was done at only one orbital phase, i.e., for a "single shot." A tight relation between mass ratio $q$ and luminosity ratio $\eta$ comes from the fact that only mainsequence stars were included in the simulation sample. More interestingly, both $\mathrm{S} / \mathrm{N}$ diagrams $(q-\mathrm{S} / \mathrm{N}$ and $\mathrm{S} / \mathrm{N}-\eta)$ show that the detection is almost independent of the $\mathrm{S} / \mathrm{N}$ as long as $\eta \gtrsim 0.3$ or equivalently $q \gtrsim 0.75$. The detection ratio is equal to the simulation average of $\sim 31 \%$. A slightly worse detection is observed at $\mathrm{S} / \mathrm{N}<20$, while the seemingly lower detection ratio at $\mathrm{S} / \mathrm{N}>80$ is a consequence of the small number of such systems in the simulation.

The detection clearly depends strongly on the system period $P$ and the difference between the projections of the orbital velocities of stars $\Delta v_{\text {orb }}$. The detection ratio is higher than $80 \%$ for 0.8 days $<P<2$ days as long as $\eta \gtrsim 0.3$. It seems that it begins to decrease at shorter periods but this again comes from the fact that very few systems were modeled in this region. For $P<30$ days, the detection is still better than $50 \%$ and falls toward 0 at roughly $1 \mathrm{yr}$. The unexpectedly high yield of binaries at short periods and $\eta<0.3$ can be explained as the 


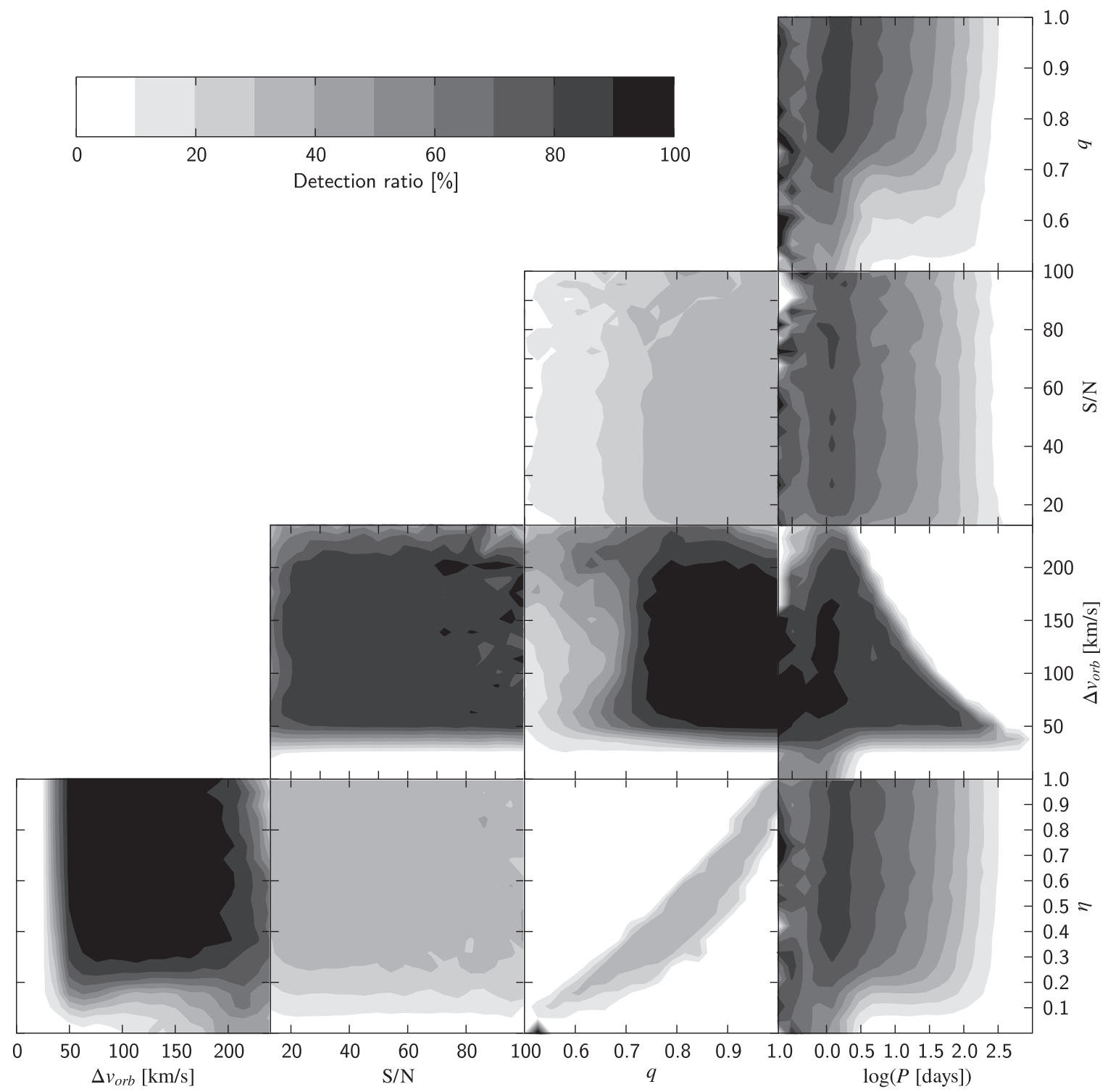

Figure 5. Ratio between detected systems and all systems in a given surface element as a function of the parameters $\Delta v_{\text {orb }}, \mathrm{S} / \mathrm{N}, q, \log P$, and $\eta$.

result of the incorrect classification of rapidly rotating cool stars as blended binaries.

The same properties can be seen on the diagrams that show the dependence of the detection ratio on the velocity difference. At $\Delta v_{\text {orb }}>40-50 \mathrm{~km} \mathrm{~s}^{-1}$, the probability of detection is greater than $90 \%$ as long as $\eta \gtrsim 0.3$. Observed spectra sometimes have a slightly lower resolving power than the model ones because of non-optimal focusing. Taking that into account it is safe to take $50 \mathrm{~km} \mathrm{~s}^{-1}$ as a lower limit. Below that value the probability for detection quickly vanishes.

The simulation shows that the target population consists mostly of binary systems with relatively well-separated spectral lines $\left(\Delta v_{\text {orb }}>50 \mathrm{~km} \mathrm{~s}^{-1}\right)$ and mass ratios close to unity $(q>0.75)$. Consequently, this implies that only shorter period binaries with $P<1 \mathrm{yr}$ will be detected, independent of the $\mathrm{S} / \mathrm{N}$, and additionally justifies the circular orbit assumption.

Since the orbital period is not measurable from a single observation, it is convenient to plot the distribution of orbital periods for all systems with a given $\Delta v_{\text {orb }}$ (shown in Figure 6). The range of orbital periods at lower velocities is greater than at higher velocities, meaning that it is possible to guess the system's period more precisely for binaries with better separated lines.

The fraction of SB2 binaries that are classified as normal single stars in the observed sample is of minor concern for studies of Galactic structure. Mistakenly treating a blended SB2 spectrum as a single one does not yield extremely wrong results because the centroid of the blended correlation peaks should be close to the systemic velocity. As for the atmospheric parameters, the similarity of both components ensures that the single star spectrum fit will still be some average of both spectra and will thus not be far from the proper solution for each component. Usually, only the rotation velocity is overestimated, but this parameter is not measurable from RAVE spectra with noteworthy precision anyway.

\section{BINARY STAR LIST}

We ran our automated classification method on the 23,321 stars in the RAVE second data release (Z08) with computed atmospheric parameters and an $\mathrm{S} / \mathrm{N}>13$. A total of 467 spectra was labeled as SB2 candidates. Upon checking all of 

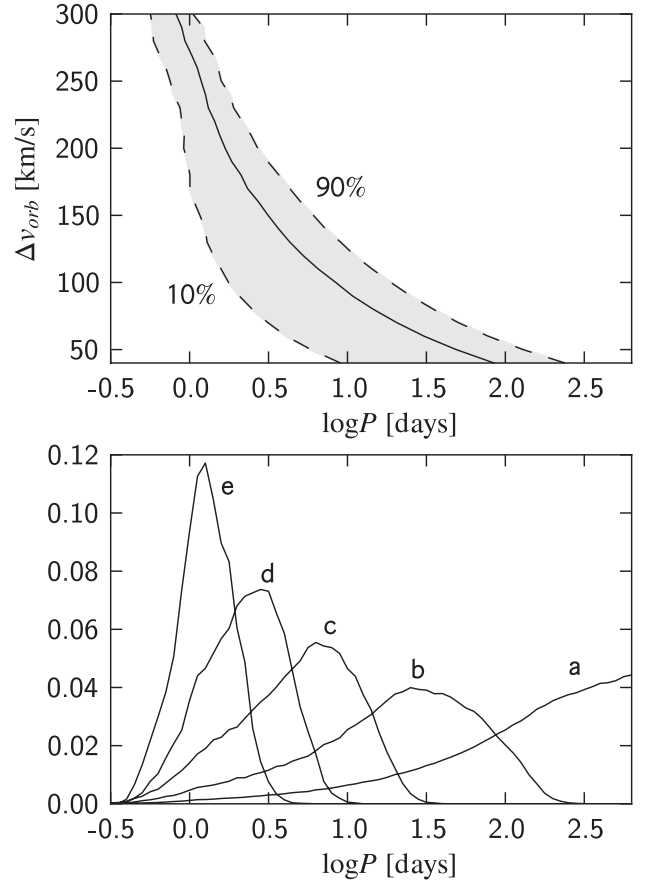

Figure 6. Shaded region in the top diagram shows the area between the 10th and 90th percentile of a distribution of line separations as a function of $\log P$. For example, at $200 \mathrm{~km} \mathrm{~s}^{-1}$ the majority of spectra (80\%) are from systems with orbital periods between 1 and 3 days. The solid line is the distribution median. The bottom diagram shows the distribution of orbital periods of systems with different line separations (a: $0-50 \mathrm{~km} \mathrm{~s}^{-1}$; b: $50-100 \mathrm{~km} \mathrm{~s}^{-1}$; c: $100-150 \mathrm{~km} \mathrm{~s}^{-1}$; d: $150-200 \mathrm{~km} \mathrm{~s}^{-1}$; e: $>200 \mathrm{~km} \mathrm{~s}^{-1}$ ) as a function of $\log P$.

them manually, only 129 of them (belonging to 123 different stars) could undoubtedly be confirmed as SB2 candidates, implying a success rate of 0.3 . For 102 of these 129 spectra, the automated classification was able to measure the separation in radial velocity, the individual temperatures, and the luminosity ratio. These data are summarized in Table 1. The first six columns of the table report the star and spectrum identification as given in the RAVE second data release (accessible through http://www.rave-survey.org), $\mathrm{S} / \mathrm{N}$ is the spectrum signal-tonoise ratio, $\Delta v_{\text {orb }}$ is the difference between red and blue Doppler-shifted lines, $\Delta v_{\text {orb }}$ error is an error of that estimate, and $\eta$ is the luminosity ratio of the less luminous to the more luminous component. $T_{\text {eff,1 }}$ and $T_{\text {eff, } 2}$ are blueshifted and redshifted component's effective temperatures, respectively. The 27 spectra for which the automated procedure could not reliably derive the separation in orbital velocity, the luminosity ratio, and temperatures of the two stars are given in Table 2.

All 22,854 spectra classified as "normal single stars" by the automated procedure were checked manually one by one, and none of them appeared to be an undetected SB2 candidate. We can conclude that the automated procedure has been successfully detecting essentially all genuine SB2 candidate spectra present in the RAVE second data release. The extra spectra picked out by the automated procedure are spurious detections caused by cool stars with wider than usual absorption lines.

The preliminary eye-inspection check carried out on the sample of RAVE second data release flagged 107 stars as potential SB2 objects. Our automatic classifications confirmed 71 of them. The remaining 36 spectra could not be confirmed even after thorough inspection and were therefore discarded.

\subsection{Binary Spectra Fitting}

The stellar parameters and radial velocities were obtained by fitting the observed binary spectra with the synthetic spectra from the library. While many methods exist for fitting single star spectra (see, e.g., Koleva et al. 2009, and references therein), none of them was reportedly used on SB2 spectra. Modeling binary spectra is more complex than modeling single star spectra for obvious reasons. The number of free parameters is more than twice as large. In our case, where we neglected all minor effects, the total number of parameters was 10: effective temperatures of both stars, their surface gravities, rotational velocities, metallicity, which was assumed to be the same for both stars, both Doppler shifts, and the luminosity ratio.

The common way of finding the best-fitting solution is to define a criterion for the goodness of fit, which is usually the sum of squares of the difference between the observed $(o)$ and modeled $(m)$ spectra, where the sum goes through all wavelength bins.

Our fitting procedure works as follows. First, the approximate Doppler shifts and luminosity ratio are obtained with the TODCOR method (Zucker \& Mazeh 1994). The parameters of both template spectra for the calculation of the CCF are always the same. With roughly known velocities and luminosity ratio, a better solution is searched for using a MultiNest algorithm (Feroz et al. 2009). This method has two big advantages over more traditional algorithms like the Nelder-Mead simplex. The final solution of the latter depends on the initial starting point on the grid of synthetic spectra. Moreover, because we are using a linear interpolation of synthetic spectra between the grid points, descending methods have a bias for finding a solution close to some grid point. Parameter space sampling methods on the other hand have a better feel for the area surrounding the global minimum and therefore a better chance of finding it.

Unfortunately, none of the tested methods gave reasonable results in performing a completely unconstrained fitting. It often happened that the method returned a solution consisting of a giant and a dwarf both with similar temperatures and a luminosity ratio close to unity. While this solution might formally be the best-fitting one, it is still not physically feasible and cannot be trusted. To overcome the problem we limited the solutions to the main-sequence stars, the same as we did in the construction of the synthetic sample for the described simulation (see Section 3.1). This simplification gave more reasonable solutions (Table 1).

The errors of the $\Delta v_{\text {orb }}$ are similar to the errors of measured radial velocities for single stars. Generally, they are larger for blended cases, where positions of lines are harder to locate and for faster rotating stars that have wider lines. Errors on individual spectral types and luminosity ratio were estimated from four reobservations. The objects T_9474_01354_1, T_8045_00353_1, T_8510_01121_1, and T_4916_01130_1 were all observed twice on different nights. The average effective temperature difference between the two solutions for the same object is equal to $130 \mathrm{~K}$ with a standard deviation of $44 \mathrm{~K}$, similar to what is returned by the MultiNest method. The errors depend on the $\mathrm{S} / \mathrm{N}$ and the separation of the lines (Doppler shifts). They are greater when the lines are closer together. The average difference of the luminosity ratio is equal to 0.08 with a standard deviation of 0.08 . Here, the dependence on line separation is much greater. When lines are separated well $\left(\Delta v_{\text {orb }} \gtrsim 100 \mathrm{~km} \mathrm{~s}^{-1}\right.$ for both measurements) the errors are smaller than 5\%, but are proportionally higher in the blended case of T_9474_01354_1 
Table 1

SB2 Candidate Spectra with Solutions

\begin{tabular}{|c|c|c|c|c|c|c|c|c|c|c|}
\hline ID & $\begin{array}{c}\text { R.A. } \\
\left({ }^{\circ}\right)\end{array}$ & $\begin{array}{c}\text { Decl. } \\
\left({ }^{\circ}\right)\end{array}$ & Obs. Date & Field Name & FNum & $\mathrm{S} / \mathrm{N}$ & $\begin{array}{c}\Delta v_{\text {orb }} \\
\left(\mathrm{km} \mathrm{s}^{-1}\right)\end{array}$ & $\begin{array}{c}\Delta v_{\text {orb }} \text { error } \\
\left(\mathrm{km} \mathrm{s}^{-1}\right)\end{array}$ & $\eta$ & $\begin{array}{c}T_{\text {eff, } 1} / T_{\text {eff }, 2} \\
(\mathrm{~K})\end{array}$ \\
\hline T8022_00693_1 & 0.39312 & -45.92950 & 20040825 & $0004 \mathrm{~m} 46$ & 80 & 42 & 83.5 & 4.6 & 0.65 & $6500 / 6200$ \\
\hline T7527_00046_1 & 3.53638 & -41.86767 & 20041022 & $0010 \mathrm{~m} 40$ & 135 & 69 & 89.2 & 4.2 & 0.39 & $6600 / 5800$ \\
\hline T8472_01036_1 & 9.15758 & -59.53453 & 20041122 & $0049 \mathrm{~m} 60$ & 49 & 46 & 170.9 & 3.8 & 0.80 & $6000 / 6200$ \\
\hline T8035_00549_1 & 18.67075 & -48.29000 & 20040824 & $0110 \mathrm{~m} 48$ & 107 & 41 & 57.6 & 8.9 & 0.65 & $5700 / 6000$ \\
\hline T8039_01118_1 & 20.92058 & -50.02906 & 20040824 & $0110 \mathrm{~m} 48$ & 132 & 43 & 131.3 & 3.2 & 0.64 & $6600 / 6300$ \\
\hline T8480_00202_1 & 21.03983 & -59.11883 & 20040827 & $0133 \mathrm{~m} 59$ & 40 & 66 & 61.2 & 6.5 & 0.79 & $6200 / 6000$ \\
\hline C0136059-154303 & 24.02483 & -15.71775 & 20041022 & $0136 \mathrm{~m} 15$ & 132 & 40 & 81.6 & 7.8 & 0.84 & $5800 / 5600$ \\
\hline T8045_00353_1* & 30.10787 & -49.07056 & 20041030 & $0213 \mathrm{~m} 49$ & 43 & 32 & 153.6 & 4.7 & 0.67 & $6600 / 7000$ \\
\hline T8045_00353_1* & 30.10787 & -49.07056 & 20041223 & $0213 m 49$ & 43 & 45 & 136.0 & 4.5 & 0.63 & $6900 / 6500$ \\
\hline T8045_00967_1* & 32.36425 & -49.55372 & 20041223 & $0213 m 49$ & 35 & 35 & 118.0 & 4.9 & 0.55 & $5600 / 6100$ \\
\hline T7554_01089_1 & 33.70387 & -41.39386 & 20041122 & $0206 \mathrm{~m} 42$ & 98 & 71 & 68.2 & 4.6 & 0.95 & $6200 / 6200$ \\
\hline T8489_00816_1 & 34.45954 & -58.06731 & 20041025 & $0212 \mathrm{~m} 56$ & 132 & 52 & 92.0 & 4.9 & 0.47 & $6000 / 5400$ \\
\hline T8055_01000_1 & 35.35021 & -50.69675 & 20041223 & $0213 m 49$ & 132 & 63 & 126.5 & 3.4 & 0.78 & $6100 / 6300$ \\
\hline T4704_00341_1 & 38.18088 & -5.46006 & 20041202 & $0238 \mathrm{~m} 05$ & 50 & 76 & 95.6 & 3.0 & 0.95 & $5900 / 5900$ \\
\hline T9155_00658_1 & 48.67517 & -71.48606 & 20041231 & $0320 \mathrm{~m} 73$ & 74 & 48 & 80.2 & 6.0 & 0.27 & $5400 / 6400$ \\
\hline T9155_01488_1 & 50.74250 & -70.78867 & 20041122 & $0320 \mathrm{~m} 73$ & 76 & 35 & 228.4 & 4.8 & 0.62 & $7000 / 6600$ \\
\hline T8493_00812_1 & 50.80117 & -52.66894 & 20041221 & $0329 \mathrm{~m} 52$ & 50 & 71 & 62.2 & 10.6 & 0.57 & $6000 / 6400$ \\
\hline T8867_00392_1 & 52.61408 & -60.71822 & 20041222 & $0336 \mathrm{~m} 62$ & 68 & 47 & 94.0 & 4.3 & 0.95 & $6200 / 6100$ \\
\hline T8063_00152_1 & 53.43492 & -47.79075 & 20040924 & $0342 \mathrm{~m} 46$ & 28 & 52 & 103.3 & 5.6 & 0.82 & $6400 / 6500$ \\
\hline T8060_01804_1 & 54.98733 & -47.20036 & 20040924 & $0342 \mathrm{~m} 46$ & 18 & 54 & 99.4 & 6.1 & 0.56 & $6400 / 5900$ \\
\hline T8867_00641_1 & 56.42312 & -60.73789 & 20041222 & $0336 \mathrm{~m} 62$ & 89 & 59 & 114.3 & 3.5 & 0.86 & $5800 / 5700$ \\
\hline C0405287-664250 & 61.36975 & -66.71392 & 20041229 & $0403 \mathrm{~m} 68$ & 78 & 34 & 154.4 & 4.9 & 0.61 & $6100 / 5700$ \\
\hline T8510_00159_1 & 71.55858 & -53.18144 & 20041122 & $0447 \mathrm{~m} 52$ & 52 & 43 & 93.5 & 6.0 & 0.47 & $5600 / 6200$ \\
\hline T8510_01121_1* & 73.24871 & -53.44589 & 20041122 & $0447 \mathrm{~m} 52$ & 132 & 43 & 116.1 & 3.4 & 0.92 & $6200 / 6300$ \\
\hline T8510_01121_1* & 73.24871 & -53.44589 & 20041123 & $0447 \mathrm{~m} 52$ & 132 & 70 & 83.8 & 3.3 & 0.92 & $6100 / 6200$ \\
\hline $\mathrm{C} 0454137-482550$ & 73.55742 & -48.43067 & 20041129 & $0449 \mathrm{~m} 46$ & 143 & 19 & 138.5 & 5.5 & 1.00 & $5800 / 5800$ \\
\hline T6469_01030_1 & 74.22913 & -26.67475 & 20041023 & $0504 \mathrm{~m} 26$ & 29 & 81 & 150.9 & 4.9 & 0.97 & $7500 / 7500$ \\
\hline T8077_00505_1 & 74.23212 & -45.94442 & 20041129 & $0449 \mathrm{~m} 46$ & 97 & 38 & 72.2 & 6.5 & 0.32 & $6400 / 5500$ \\
\hline T7053_00933_1 & 77.98013 & -36.82828 & 20050129 & $0516 \mathrm{~m} 37$ & 61 & 43 & 144.6 & 3.8 & 0.41 & $6200 / 5500$ \\
\hline T7594_00902_1 & 79.94879 & -42.58228 & 20041025 & $0520 \mathrm{~m} 42$ & 18 & 54 & 121.6 & 6.3 & 0.22 & $5200 / 6400$ \\
\hline T6501_00207_1 & 84.38304 & -29.27319 & 20050128 & $0535 \mathrm{~m} 29$ & 94 & 29 & 110.9 & 4.3 & 0.42 & $5700 / 6400$ \\
\hline C0538506-401127 & 84.71121 & -40.19092 & 20050221 & $0549 \mathrm{~m} 40$ & 46 & 34 & 125.5 & 5.3 & 0.67 & $6100 / 5700$ \\
\hline T9386_01431_1 & 84.90012 & -79.97753 & 20050128 & $0609 \mathrm{~m} 80$ & 40 & 40 & 251.4 & 6.4 & 0.42 & $7300 / 6400$ \\
\hline T8891_03299_1 & 85.22208 & -67.12572 & 20041222 & $0517 \mathrm{~m} 65$ & 130 & 58 & 135.2 & 4.1 & 0.94 & $6800 / 6900$ \\
\hline T9163_00869_1 & 88.10375 & -68.15956 & 20041229 & $0614 \mathrm{~m} 68$ & 45 & 32 & 73.1 & 7.7 & 0.79 & $5400 / 5600$ \\
\hline T7606_01468_1 & 88.90250 & -42.68181 & 20041129 & $0549 \mathrm{~m} 40$ & 143 & 55 & 122.3 & 6.2 & 0.23 & $6500 / 5300$ \\
\hline T8894_00627_1 & 95.01929 & -60.68039 & 20041122 & $0611 \mathrm{~m} 63$ & 84 & 41 & 140.4 & 3.4 & 0.98 & $6500 / 6400$ \\
\hline T8898_00763_1 & 97.86787 & -63.53539 & 20050129 & $0611 \mathrm{~m} 63$ & 120 & 56 & 169.2 & 6.5 & 0.32 & $6300 / 7600$ \\
\hline OCL00153_1457872 & 114.89513 & -16.35411 & 20041202 & $0739 \mathrm{~m} 14$ & 143 & 37 & 97.5 & 5.2 & 0.22 & $6600 / 5400$ \\
\hline T5491_00836_1 & 156.59321 & -8.73600 & 20040510 & $1025 \mathrm{~m} 08$ & 118 & 62 & 166.1 & 4.4 & 0.57 & $6400 / 6000$ \\
\hline C1040349-123408 & 160.14554 & -12.56892 & 20050221 & $1042 \mathrm{~m} 11$ & 32 & 19 & 124.4 & 7.0 & 0.53 & $6000 / 5500$ \\
\hline T4916_01130_1* & 160.17733 & -4.73567 & 20041231 & $1040 \mathrm{~m} 04$ & 136 & 59 & 136.6 & 3.9 & 0.54 & $5300 / 5800$ \\
\hline T4916_01130_1* & 160.17733 & -4.73567 & 20050131 & $1040 \mathrm{~m} 04$ & 136 & 52 & 141.1 & 4.3 & 0.49 & $5600 / 5100$ \\
\hline T0255_00172_1 & 163.44150 & 0.70903 & 20050331 & $1101 \mathrm{~m} 01$ & 59 & 67 & 165.0 & 4.3 & 0.52 & $6500 / 6000$ \\
\hline T6076_01000_1 & 163.57917 & -17.06961 & 20050301 & $1101 \mathrm{~m} 15$ & 19 & 64 & 64.9 & 7.4 & 0.90 & $5700 / 5800$ \\
\hline T6661_01196_1 & 169.15446 & -29.64906 & 20040628 & $1114 \mathrm{~m} 29$ & 107 & 68 & 56.1 & 7.7 & 0.70 & $5900 / 5600$ \\
\hline C1153324-145540 & 178.38538 & -14.92797 & 20040508 & $1200 \mathrm{~m} 15$ & 49 & 20 & 82.7 & 5.5 & 0.85 & $6400 / 6300$ \\
\hline C1154492-321905 & 178.70504 & -32.31819 & 20050130 & $1204 \mathrm{~m} 33$ & 53 & 22 & 75.8 & 5.3 & 0.57 & $5200 / 5600$ \\
\hline T7235_00510_1 ${ }^{\mathrm{b}}$ & 180.36558 & -32.22625 & 20050130 & $1204 \mathrm{~m} 33$ & 65 & 43 & 150.5 & 4.6 & 0.82 & $6200 / 6000$ \\
\hline T5519_01279_1 & 181.30896 & -9.36233 & 20040704 & $1200 \mathrm{~m} 09$ & 117 & 79 & 161.8 & 4.5 & 0.29 & $7600 / 6500$ \\
\hline T7245_00609_1 & 185.15921 & -35.80486 & 20040628 & $1232 \mathrm{~m} 34$ & 23 & 29 & 131.0 & 4.8 & 0.89 & $6400 / 6500$ \\
\hline T6690_01250_1 & 187.25942 & -26.25542 & 20050226 & $1226 \mathrm{~m} 28$ & 81 & 72 & 156.9 & 5.0 & 0.88 & $6600 / 6500$ \\
\hline C1239461-315947 & 189.94237 & -31.99644 & 20040628 & $1232 \mathrm{~m} 34$ & 89 & 36 & 151.5 & 8.8 & 0.42 & $6100 / 5400$ \\
\hline T6709_00114_1 & 193.29750 & -29.43997 & 20040704 & $1252 \mathrm{~m} 28$ & 123 & 77 & 51.7 & 6.6 & 0.99 & $6100 / 6100$ \\
\hline $\mathrm{C} 1300428-055402$ & 195.17838 & -5.90081 & 20040629 & $1252 \mathrm{~m} 05$ & 115 & 20 & 110.1 & 4.4 & 0.97 & $5100 / 5200$ \\
\hline T6717_00250_1 & 201.29679 & -24.86047 & 20050221 & $1321 \mathrm{~m} 22$ & 146 & 58 & 61.9 & 7.3 & 0.64 & $6100 / 5700$ \\
\hline T7270_00030_1 & 206.95521 & -33.42233 & 20040627 & $1353 \mathrm{~m} 32$ & 17 & 78 & 133.7 & 4.0 & 0.92 & $7200 / 7400$ \\
\hline T6148_00058_1 & 208.41325 & -21.83800 & 20050301 & $1345 \mathrm{~m} 21$ & 120 & 70 & 74.2 & 5.3 & 0.98 & $5500 / 5500$ \\
\hline T6148_00150_1 & 208.46092 & -22.48617 & 20040529 & $1345 \mathrm{~m} 21$ & 129 & 70 & 154.8 & 3.4 & 0.71 & $6200 / 6500$ \\
\hline T6142_00026_1 & 212.89283 & -17.22064 & 20040705 & $1408 \mathrm{~m} 19$ & 81 & 40 & 59.5 & 10.4 & 0.41 & $5300 / 6000$ \\
\hline T7282_00604_1 & 214.94333 & -30.97186 & 20040507 & $1419 \mathrm{~m} 30$ & 12 & 64 & 123.5 & 5.8 & 0.40 & $6000 / 6800$ \\
\hline T4999_01159_1 & 224.55354 & -6.32244 & 20050320 & $1456 \mathrm{~m} 05$ & 90 & 97 & 168.4 & 6.4 & 0.34 & $6400 / 7800$ \\
\hline C1514052-223119 & 228.52179 & -22.52217 & 20050321 & $1524 \mathrm{~m} 21$ & 32 & 18 & 188.2 & 7.9 & 0.92 & $6600 / 6700$ \\
\hline 154550 & 257.02162 & -41.25581 & 20040924 & $1716 \mathrm{~m} 42$ & 60 & 70 & 83.5 & 3.2 & 0.70 & $6100 / 5900$ \\
\hline TYC_6269-14-1 & 274.46946 & -17.31033 & 20040925 & $1822 \mathrm{~m} 16$ & 15 & 47 & 106.9 & 6.2 & 0.43 & $9400 / 7500$ \\
\hline
\end{tabular}


Table 1

(Continued)

\begin{tabular}{|c|c|c|c|c|c|c|c|c|c|c|}
\hline ID & $\begin{array}{c}\text { R.A. } \\
\left({ }^{\circ}\right)\end{array}$ & $\begin{array}{c}\text { Decl. } \\
\left(^{\circ}\right)\end{array}$ & Obs. Date & Field Name & FNum & $\mathrm{S} / \mathrm{N}$ & $\begin{array}{c}\Delta v_{\text {orb }} \\
\left(\mathrm{km} \mathrm{s}^{-1}\right)\end{array}$ & $\begin{array}{c}\Delta v_{\text {orb }} \text { error } \\
\left(\mathrm{km} \mathrm{s}^{-1}\right)\end{array}$ & $\eta$ & $\begin{array}{c}T_{\text {eff, } 1} / T_{\text {eff, } 2} \\
(\mathrm{~K})\end{array}$ \\
\hline T9458_00473_1 & 285.11504 & -76.27161 & 20040825 & $1929 \mathrm{~m} 76$ & 43 & 98 & 150.0 & 3.1 & 0.48 & $6900 / 6300$ \\
\hline T9458_00642_1 & 289.45371 & -75.53994 & 20040502 & $1929 \mathrm{~m} 76$ & 61 & 53 & 88.3 & 4.9 & 0.49 & $6800 / 6200$ \\
\hline C2002522-265300 & 300.71754 & -26.88344 & 20040530 & $2008 \mathrm{~m} 28$ & 66 & 36 & 95.2 & 4.7 & 0.29 & $6000 / 5000$ \\
\hline T6327_00704_1 & 302.40129 & -21.45864 & 20040530 & $2016 \mathrm{~m} 23$ & 64 & 72 & 210.8 & 9.8 & 0.80 & $8500 / 8000$ \\
\hline T8776_00144_1 & 303.03658 & -52.97231 & 20040825 & $2018 \mathrm{~m} 53$ & 50 & 46 & 88.4 & 4.2 & 0.83 & $5700 / 5900$ \\
\hline T6340_00410_1 & 305.15842 & -22.04008 & 20040529 & $2016 \mathrm{~m} 23$ & 83 & 38 & 71.5 & 5.0 & 0.43 & $5600 / 6300$ \\
\hline T6333_00993_1 & 306.12083 & -16.89067 & 20040529 & $2024 \mathrm{~m} 18$ & 68 & 51 & 104.3 & 4.3 & 0.28 & $7600 / 6200$ \\
\hline T5766_01122_1 & 306.83275 & -14.16467 & 20040629 & $2034 \mathrm{~m} 12$ & 22 & 50 & 147.8 & 4.6 & 0.89 & $6000 / 6100$ \\
\hline T9308_00697_1 & 307.64242 & -68.24917 & 20040902 & $2037 \mathrm{~m} 70$ & 71 & 35 & 85.2 & 8.5 & 0.83 & $5300 / 5500$ \\
\hline C2033361-142800 & 308.40050 & -14.46683 & 20040531 & $2034 \mathrm{~m} 12$ & 4 & 24 & 101.2 & 6.0 & 0.34 & $6000 / 5200$ \\
\hline T6330_00204_1 & 308.44429 & -16.76408 & 20040531 & $2024 \mathrm{~m} 18$ & 98 & 34 & 69.7 & 5.8 & 0.45 & $6300 / 5700$ \\
\hline T7468_01360_1 & 308.69225 & -36.47119 & 20040508 & $2028 \mathrm{~m} 35$ & 134 & 63 & 73.7 & 6.5 & 0.35 & $6600 / 5800$ \\
\hline T9316_00774_1 & 308.77992 & -71.66714 & 20040629 & $2037 \mathrm{~m} 70$ & 144 & 40 & 245.5 & 4.0 & 0.83 & $7400 / 7100$ \\
\hline T9312_00707_1 & 309.10854 & -70.08231 & 20040629 & $2037 \mathrm{~m} 70$ & 102 & 39 & 71.3 & 4.6 & 0.81 & $6100 / 6200$ \\
\hline T7468_00041_1 & 309.32412 & -36.77611 & 20040706 & $2028 \mathrm{~m} 35$ & 131 & 27 & 67.1 & 10.6 & 0.41 & $6400 / 5900$ \\
\hline T9329_00060_1 & 313.35037 & -71.83719 & 20040902 & $2037 \mathrm{~m} 70$ & 131 & 71 & 73.3 & 5.8 & 0.68 & $6300 / 6000$ \\
\hline T6345_01096_1 & 315.91971 & -15.25700 & 20040627 & $2058 \mathrm{~m} 13$ & 136 & 92 & 89.3 & 3.6 & 0.58 & $5900 / 6300$ \\
\hline C2122581-243821 & 320.74225 & -24.63917 & 20040531 & $2133 \mathrm{~m} 24$ & 41 & 21 & 150.4 & 4.2 & 0.64 & $5700 / 5400$ \\
\hline T8806_00524_1 & 321.79408 & -54.34042 & 20040924 & $2136 \mathrm{~m} 53$ & 38 & 45 & 178.3 & 4.8 & 0.54 & $5700 / 5200$ \\
\hline T9474_01354_1* & 322.07942 & -75.95464 & 20041003 & $2106 \mathrm{~m} 75$ & 114 & 39 & 85.1 & 4.1 & 0.72 & $5600 / 5300$ \\
\hline T9474_01354_1* & 322.07942 & -75.95464 & 20041025 & $2106 \mathrm{~m} 75$ & 114 & 48 & 62.6 & 9.4 & 0.50 & $5100 / 5600$ \\
\hline C2142493-091026 & 325.70562 & -9.17411 & 20040628 & $2133 \mathrm{~m} 08$ & 117 & 22 & 78.6 & 4.2 & 0.90 & $5500 / 5600$ \\
\hline T8812_00684_1 & 326.68233 & -55.19394 & 20040924 & $2136 \mathrm{~m} 53$ & 134 & 36 & 116.8 & 4.3 & 0.69 & $6600 / 6300$ \\
\hline T7495_00875_1 & 330.05267 & -35.33600 & 20040607 & $2153 \mathrm{~m} 35$ & 115 & 30 & 119.4 & 5.2 & 0.74 & $5400 / 5200$ \\
\hline T7495_01110_1 & 331.44988 & -35.75942 & 20040607 & $2153 \mathrm{~m} 35$ & 117 & 59 & 118.4 & 3.2 & 0.76 & $5600 / 5400$ \\
\hline T5810_00770_1 & 335.97663 & -13.81914 & 20040628 & $2216 \mathrm{~m} 13$ & 114 & 66 & 86.0 & 3.0 & 0.56 & $6500 / 6100$ \\
\hline T7498_00637_1 & 339.87038 & -31.50889 & 20040825 & $2247 \mathrm{~m} 33$ & 60 & 43 & 87.6 & 5.2 & 0.70 & $6200 / 6000$ \\
\hline C2240261-515558 & 340.10892 & -51.93294 & 20040827 & $2258 \mathrm{~m} 52$ & 43 & 34 & 114.0 & 4.8 & 0.46 & $5400 / 6000$ \\
\hline T8450_00444_1 & 341.65946 & -48.38072 & 20040824 & $2239 \mathrm{~m} 47$ & 117 & 90 & 51.3 & 6.7 & 0.69 & $6400 / 6200$ \\
\hline T8824_01026_1 & 344.63675 & -54.00822 & 20040827 & $2258 \mathrm{~m} 52$ & 149 & 46 & 146.7 & 3.6 & 0.83 & $5800 / 5600$ \\
\hline T5827_00145_1 & 348.04021 & -13.04881 & 20040627 & $2314 \mathrm{~m} 11$ & 21 & 82 & 140.6 & 3.4 & 0.89 & $6100 / 6000$ \\
\hline C2317138-020625 & 349.30762 & -2.10719 & 20040626 & $2313 \mathrm{~m} 03$ & 86 & 28 & 131.7 & 5.7 & 0.63 & $5900 / 5600$ \\
\hline T6399_00230_1 & 350.09596 & -19.02089 & 20041031 & $2321 \mathrm{~m} 20$ & 67 & 32 & 82.8 & 3.9 & 0.99 & $5500 / 5500$ \\
\hline C2348525-211646 & 357.21913 & -21.27961 & 20041101 & $2342 \mathrm{~m} 23$ & 87 & 20 & 92.8 & 8.2 & 0.46 & $5800 / 6400$ \\
\hline T9127_00460_1 & 358.00408 & -60.74483 & 20040824 & $0004 m 59$ & 17 & 46 & 72.4 & 6.6 & 0.51 & $6400 / 6000$ \\
\hline C2352470-522138 & 358.19604 & -52.36069 & 20041025 & $2351 \mathrm{~m} 52$ & 62 & 29 & 86.8 & 6.9 & 0.49 & $5800 / 6400$ \\
\hline C2353295-442121 & 358.37329 & -44.35589 & 20041030 & $2339 \mathrm{~m} 43$ & 126 & 18 & 102.7 & 7.1 & 0.39 & $5200 / 6000$ \\
\hline
\end{tabular}

Notes. Spectra IDs marked with asterisks were observed twice. The second observation of object T8045_00967_1 is listed in Table 2.

a A known binary star, Heintz (1992).

${ }^{\mathrm{b}}$ DZ Hya, eclipsing binary of the Algol type.

(more than 20\%). This holds true only if the main-sequence assumptions can be justified.

\subsection{Notes on Individual Objects}

While the spectra listed in Table 1 do not show any special features, some of those in Table 2 do and can be grouped together.

\subsubsection{Ca II Emission}

Six different objects (one observed twice) show chromospheric emission in the Ca II lines, similarly to what is sometimes observed in the same lines of single stars. All iron lines, especially the ones at $8515 \AA, 8647 \AA$, and $8688 \AA$, show duplicity (Figure 7), although they are harder to identify in some of the noisier spectra. All seven spectra are consistent with G-type stars. The Ca II triplet lines of both components are too shallow, which indicates that some of the flux originates from emission processes. These stars are probably members of the RS CVn group of binaries with active chromospheres. G spectral types and emission cores in the Ca II lines are their distinctive features, with the active chromospheres powered by intense magnetic fields generated by tidal interaction between the two components (Foing et al. 1989; Eker et al. 2008). Object T5844_00340_1 was observed twice in the span of one month and shows changes in the positions of lines. Iron lines in C0232159_055451 are hardly observable, but shapes of the $\mathrm{Ca}$ II triplet lines are consistent with other objects of this kind.

\subsubsection{Contact Binaries}

Another group of morphologically similar spectra are potential new contact binary stars, shown in the middle plot of Figure 7. Four of the stars, BE Scl, DX Tuc, V1054 Cen, and DY Cet, are known W UMa type eclipsing binaries. Fifth, IV Pav, is still listed as an RR Lyr type variable in the SIMBAD database, but it was already suggested from Hipparcos observations that it might be a close binary (Solano et al. 1997; Fernley et al. 1998). Consistent shapes of spectra with very wide Ca II lines that are still clearly separable indicate a small semimajor axis and co-rotation of both stars. The other seven spectra in 
Table 2

Peculiar and Low S/N SB2 Candidate Spectra

\begin{tabular}{|c|c|c|c|c|c|c|c|}
\hline ID & $\begin{array}{c}\text { R.A. } \\
\left({ }^{\circ}\right)\end{array}$ & $\begin{array}{c}\text { Decl. } \\
\left({ }^{\circ}\right)\end{array}$ & Obs. Date & Field Name & FNum & $\mathrm{S} / \mathrm{N}$ & Comment \\
\hline T5844_00340_1* & 1.20621 & -21.69836 & 20040923 & $0014 \mathrm{~m} 21$ & 35 & 37 & Ca II emission \\
\hline T5844_00340_1* & 1.20621 & -21.69836 & 20041024 & $0014 \mathrm{~m} 21$ & 34 & 33 & Ca II emission \\
\hline C0028572-081237 & 7.23858 & -8.21047 & 20040629 & $0030 \mathrm{~m} 06$ & 2 & 24 & Contact? \\
\hline ТC0102115-380905 & 15.54792 & -38.15161 & 20040924 & $0103 \mathrm{~m} 37$ & 8 & 29 & Contact? \\
\hline C0102282-105314 & 15.61779 & -10.88733 & 20041202 & $0053 \mathrm{~m} 11$ & 109 & 19 & Low $\mathrm{S} / \mathrm{N}$ \\
\hline T5275_00027_1 & 15.86400 & -12.17308 & 20041202 & $0053 \mathrm{~m} 11$ & 124 & 83 & Contact? \\
\hline T6427_00323_1 & 20.38729 & -29.13131 & 20040825 & $0111 \mathrm{~m} 27$ & 132 & 64 & BE Scl, W UMa type \\
\hline T5855_00622_1 & 25.11900 & -16.10931 & 20041023 & $0136 \mathrm{~m} 15$ & 134 & 63 & Ca II emission \\
\hline T8045_00967_1* & 32.36425 & -49.55372 & 20041030 & $0213 \mathrm{~m} 49$ & 35 & 15 & Low $\mathrm{S} / \mathrm{N}$ \\
\hline C0232159-055451 & 38.06658 & -5.91428 & 20041202 & $0238 \mathrm{~m} 05$ & 44 & 24 & Ca II emission \\
\hline T5291_00361_1 & 39.63825 & -14.29908 & 20040902 & $0231 \mathrm{~m} 12$ & 131 & 99 & DY Cet, W UMa type \\
\hline C0238506-130910 & 39.71104 & -13.15300 & 20040902 & $0231 \mathrm{~m} 12$ & 116 & 34 & Ca II emission \\
\hline C0241430-062149 & 40.42942 & -6.36364 & 20041202 & $0238 \mathrm{~m} 05$ & 123 & 32 & Contact? \\
\hline T8093_00960_1 & 79.82367 & -49.64683 & 20041101 & $0523 \mathrm{~m} 48$ & 22 & 16 & Low $\mathrm{S} / \mathrm{N}$ \\
\hline T8905_00616_1 & 91.98046 & -66.36028 & 20041221 & $0614 \mathrm{~m} 68$ & 72 & 77 & Contact? \\
\hline T7216_00384_1 & 174.61763 & -30.29139 & 20040627 & $1138 \mathrm{~m} 31$ & 74 & 74 & HD 101167 , triple \\
\hline C1230542-330934 & 187.72588 & -33.15950 & 20040531 & $1232 \mathrm{~m} 34$ & 53 & 25 & Low $\mathrm{S} / \mathrm{N}$ \\
\hline T6103_01209_1 & 188.19242 & -16.43886 & 20040706 & $1223 \mathrm{~m} 16$ & 110 & 63 & Contact? \\
\hline T7246_01161_1 & 188.20421 & -35.69497 & 20040531 & $1232 \mathrm{~m} 34$ & 143 & 47 & V1054 Cen, W UMa type \\
\hline C1245296-302913* & 191.37354 & -30.48700 & 20040529 & $1252 \mathrm{~m} 28$ & 15 & 24 & Low $\mathrm{S} / \mathrm{N}$ \\
\hline C1248186-101332 & 192.07758 & -10.22578 & 20050322 & $1246 \mathrm{~m} 11$ & 82 & 29 & Ca II emission \\
\hline T6130_00031_1 & 205.69717 & -18.88536 & 20050301 & $1345 \mathrm{~m} 21$ & 72 & 73 & Contact? \\
\hline O00337_2343045 & 207.45533 & -62.23206 & 20040728 & $1352 \mathrm{~m} 62$ & 80 & 47 & Corrupted wavelengths \\
\hline T6159_00232_1 & 223.16154 & -18.37425 & 20040501 & $1452 \mathrm{~m} 20$ & 76 & 69 & Ca II emission \\
\hline T9316_00114_1 & 308.68708 & -72.61533 & 20040902 & $2037 \mathrm{~m} 70$ & 4 & 48 & IV Pav, RRLyr type, contact? \\
\hline C2256145-342140 & 344.06067 & -34.36114 & 20040825 & $2247 \mathrm{~m} 33$ & 129 & 16 & Low $\mathrm{S} / \mathrm{N}$ \\
\hline T9130_01530_1 & 359.33967 & -64.24322 & 20040902 & $0009 \mathrm{~m} 65$ & 60 & 67 & DX Tuc, W UMa type \\
\hline
\end{tabular}

Notes. The wavelength calibration of the spectrum of O00337_2343045 is wrong, but it can nevertheless still be unambiguously confirmed as an SB2 candidate. The second observation of C1245296_302913 is not listed since its spectrum could not be recognized as a potential SB2.

the diagram are very similar to those of known contact binary spectra. It is likely that all of them are close binary systems.

\subsection{3. $H D 101167$}

The spectrum of HD 101167 is particularly interesting since it shows three components (bottom plot in Figure 7). Three Ca II lines as well as the $8674 \AA \mathrm{Fe}$ I and $8442 \AA$ O I lines are clearly triple. The best-fitting solution suggests that the system consists of two early-type $\mathrm{G}$ stars at roughly $\pm 100 \mathrm{~km} \mathrm{~s}^{-1}$ and one late-type $\mathrm{G}$ star at $\sim 0 \mathrm{~km} \mathrm{~s}^{-1}$. A proposed explanation is a hierarchically ordered triple system with a fainter and cooler star orbiting around the center of mass of a closer system of two hotter components. HD 101167 is extraordinarily similar to the triple G-type system CN Lyn, already studied over the RAVE wavelength range by Munari (2003).

\section{DISCUSSION AND CONCLUSION}

This paper presents a method to identify SB2 candidate spectra among a sample of stellar spectra covering the near-IR Ca II triplet region at a resolution of $R \sim 7500$, and a list of discovered binaries in the latest RAVE data release. From repeated observations of the same objects it is also possible to detect SB1 spectra, which will be discussed in a forthcoming paper. Joining both types will give a basis for the population study of binary stars in the RAVE survey.

The classification method based on the $\mathrm{CCF}$ proved to work efficiently for the detection of potential double-lined spectroscopic binaries. The gain of discovered SB2 binary candidates in the selected DR2 sample of the RAVE survey was small compared to other higher resolution surveys, but still significant. Out of 25,850 examined spectra, we discovered $123(0.47 \%)$ unique SB2 candidate spectra. Only seven were previously known. If we extrapolate this ratio to the entire RAVE sample and assume that the majority of candidate spectra indeed belong to binaries, more than 2000 new SB2 binaries of different kinds will be discovered in the upcoming data releases and will be treated in forthcoming papers. The list of discovered binaries also includes several unusual objects. Among them are a few binary spectra that show signs of chromospheric activity, several close or contact binaries, and a triple system HD 101167.

The detection simulation performed on a large representative sample of synthetic binary spectra showed that the majority of short-period binaries ( 0.8 days $<P<2$ days) are discovered if the luminosity ratio is $\gtrsim 0.3$. Assuming that most of the binaries lie along the main sequence the luminosity ratio translates to a mass ratio of $\gtrsim 0.75$. At longer periods the detection rate gets progressively smaller, and finally at around $1 \mathrm{yr}$ the probability for detection vanishes.

Fitting the selected sample of binary spectra proved that it is possible to measure Doppler shifts with errors comparable to the RV errors of single stars. The solutions for temperatures and surface gravities from which we derived spectral types were unreliable when fitting the spectra completely unconstrained. We got better results with the assumed main-sequence solutions for both components. There is still room for improvement. Using a more sophisticated model than only a mainsequence assumption could provide even more reliable information on spectral types and also on chemical composition of binaries. 


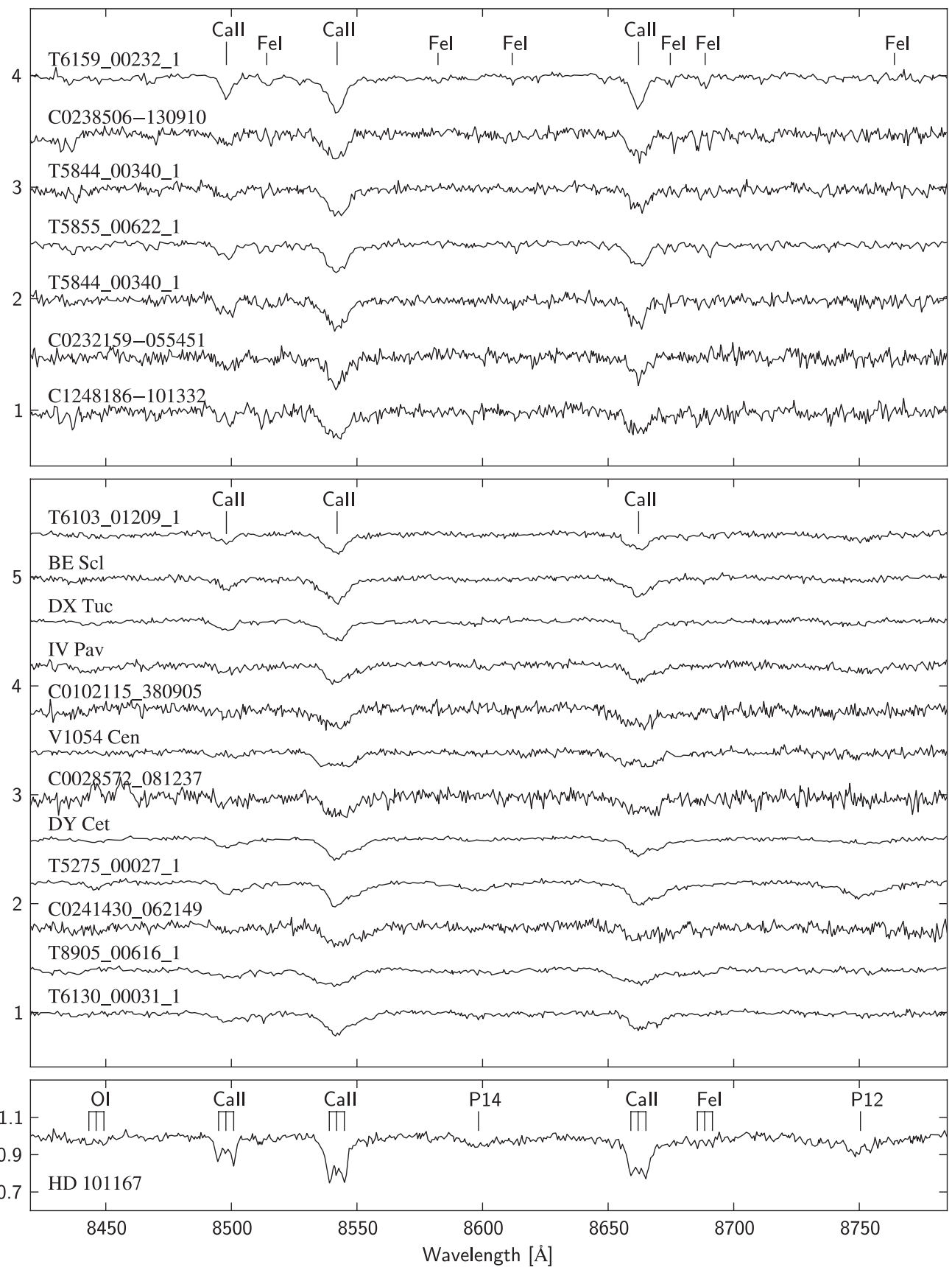

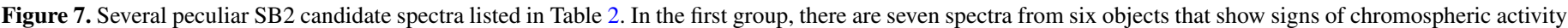

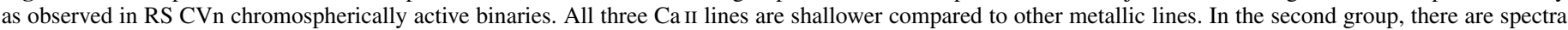

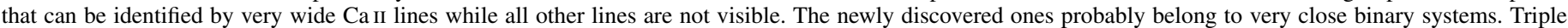

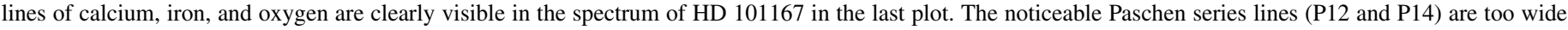
and are visible as a single blended line.

Other comparable large-scale spectroscopic surveys are the Geneva-Copenhagen (GC) survey (Nordström et al. 1994) and the Sloan Digital Sky Survey. While the latter is not suited for SB2 observation due to a low resolving power, the former was particularly successful with binary detection. Nordström et al. reported that out of $\sim 14,000$ stars around $34 \%$ were either visual or spectroscopic binaries and $510(\sim 3.6 \%)$ of them showed double lines. The detection of such a high fraction of binaries was possible because of several repeated observations of each object in the longer time span and because of the better resolution of the CORAVEL spectrometer. We ran the detection simulation with the same distribution of re- observations as in the GC survey and the efficiency of SB2 detection was approximately 50\% higher than in the case of only one observation per object. Unfortunately, the number of discovered SB2 spectra in the GC survey with velocity difference greater than $\sim 50 \mathrm{~km} \mathrm{~s}^{-1}$ is not available so a direct comparison of efficiency between the GC and RAVE surveys cannot be done.

The described classification method could also be useful for the forthcoming Gaia mission. The spectral data provided by the mission will be very similar to the RAVE data, meaning the method will be applicable without any significant modifications. The enormous scale of Gaia observations and the fact that each 
object will be observed many times during the lifetime of the mission will yield a large amount of potential new SB2 objects.

We thank the referee, David Latham, for helpful comments that improved the clarity of the text. Funding for RAVE has been provided by the Anglo-Australian Observatory, the Astrophysical Institute Potsdam, the Australian National University, the Australian Research Council, the French National Research Agency, the German Research Foundation, the Istituto Nazionale di Astrofisica at Padova, The Johns Hopkins University, the W. M. Keck Foundation, the Macquarie University, The Netherlands Research School for Astronomy, the Natural Sciences and Engineering Research Council of Canada, the Slovenian Research Agency, the Swiss National Science Foundation, the Science \& Technology Facilities Council of the UK, Opticon, Strasbourg Observatory, and the Universities of Groningen, Heidelberg, and Sydney.

\section{REFERENCES}

Bate, M. R. 2009, MNRAS, 392, 590

Boeche, C., et al. 2010, A\&A, submitted

Breddels, M. A., et al. 2010, A\&A, 511, 90

Duquennoy, A., \& Mayor, M. 1991, A\&A, 1248, 485

Eggleton, P. P. 1983, ApJ, 268, 368

Eker, Z., et al. 2008, MNRAS, 389, 1722

Fernley, J., et al. 1998, A\&A, 330, 515

Feroz, F., Hobson, M. P., \& Bridges, M. 2009, MNRAS, 398, 1601
Foing, B. H., Crivellari, L., Vladilo, G., Rebolo, R., \& Beckman, J. E. 1989, A\&AS, 80, 189

Glebocki, R., \& Stawikowski, A. 2000, Acta Astron., 50, 509

Heintz, W. D. 1992, ApJS, 83, 351

Koleva, M., Prugniel, P., Bouchard, A., \& Wu, Y. 2009, A\&A, 501, 1269

Latham, D. W. 2003, in ASP Conf. Ser. 294, Scientific Frontiers in Research on Extrasolar Planets, ed. D. Deming \& S. Seager (San Francisco, CA: ASP), 409

Malkov, O. Y., Oblak, E., Snegireva, E. A., \& Torra, J. 2006, A\&A, 446, 785

Munari, U. 2003, in ASP Conf. Ser. 298, Gaia Spectroscopy: Science and Technology, ed. U. Munari (San Francisco, CA: ASP), 55

Munari, U., Dallaporta, S., Siviero, A., Soubiran, C., Fiorucci, M., \& Girard, P. 2004, A\&A, 418, L31

Munari, U., Sordo, R., Castelli, F., \& Zwitter, T. 2005, A\&A, 442, 1127

Munari, U., et al. 2008, A\&A, 488, 969

Munari, U., et al. 2009, A\&A, 503, 511

Nordström, B., et al. 1994, A\&A, 418, 989

Seabroke, G. M., et al. 2008, MNRAS, 384, 11

Siebert, A., et al. 2008, MNRAS, 391, 793

Smith, M. C., et al. 2007, MNRAS, 379, 755

Solano, E., Garrido, R., Fernley, J., \& Barnes, T. G. 1997, A\&AS, 125, 321

Steinmetz, M., et al. 2006, AJ, 132, 1645

Tomasella, L., Munari, U., Siviero, A., Cassisi, S., Dallaporta, S., Zwitter, T., \& Sordo, R. 2008, A\&A, 480, 465

Torres, G., Andersen, J., \& Giménez, A. 2009, A\&AR, 18, 67

van Leeuwen, F. 2007, in Astrophys. Space Sci. Libr. 350, Hipparcos: the New Reduction of the Raw Data

Veltz, L., et al. 2008, A\&A, 480, 753

Yi, S., et al. 2001, ApJS, 136, 417

Zucker, S., \& Mazeh, T. 1994, ApJ, 420, 806

Zwitter, T., Castelli, F., \& Munari, U. 2004, A\&A, 417, 1055

Zwitter, T., et al. 2008, AJ, 136, 421 (Z08) 